\title{
Transcriptional Profiling and Dynamical Regulation Analysis Identify Potential Kernel Target Genes of SCYL1-BP1 in HEK293T Cells
}

\author{
Yang Wang ${ }^{1}$, Xiaomei Chen ${ }^{1}$, Xiaojing Chen ${ }^{1}$, Qilong Chen ${ }^{2, *}$, and Keke Huo ${ }^{1, *}$
}

SCYL1-BP1 is thought to function in the p53 pathway through Mdm2 and hPirh2, and mutations in SCYL1-BP1 are associated with premature aging syndromes such as Geroderma Osteodysplasticum; however, these mechanisms are unclear. Here, we report significant alterations in miRNA expression levels when SCYL1-BP1 expression was inhibited by RNA interference in HEK293T cells. We functionally characterized the effects of potential kernel miRNAtarget genes by miRNA-target network and protein-protein interaction network analysis. Importantly, we showed the diminished SCYL1-BP1 dramatically reduced the expression levels of EEA1, BMPR2 and BRCA2 in HEK293T cells. Thus, we infer that SCYL1-BP1 plays a critical function in HEK293T cell development and directly regulates miRNAtarget genes, including, but not limited to, EEA1, BMPR2, and $B R C A 2$, suggesting a new strategy for investigating the molecular mechanism of SCYL1-BP1.

\section{INTRODUCTION}

The cDNA encoding SCY1-like 1 binding protein 1 (SCYL1-BP1; accession number: NM_152281) was originally cloned in our laboratory from a human fetal liver cDNA library. SCYL1-BP1 is soluble, highly conserved and widely expressed in many tissues. SCYL1-BP1 can interact with SCY1-like protein 1 (SCYL1)(Di et al., 2003) and RING finger and CHY zinc finger domaincontaining protein 1 (hPirh2) (Yan et al., 2010a; Zhang et al., 2005). SCYL1-BP1 co-localizes with SCYL1 and may be involved in mitosis-related cellular function via binding SCYL1 (Di et al., 2003). In addition, SCYL1-BP1 was reported to be a ubiquitination substrate of hPirh2 and can induce Double minute 2 protein(Mdm2) self-ubiquitination by interacting with $\mathrm{Mdm} 2$

\footnotetext{
${ }^{1}$ State Key Laboratory of Genetic Engineering, Institute of Genetics, School of Life Sciences, Fudan University, Shanghai 200433, China, ${ }^{2}$ Research Center for TCM Complexity System, Shanghai University of TCM, Shanghai 201203, China

*Correspondence: cqlw1975@126.com (QC); kkhuo@fudan.edu.cn (KH)
}

Received 30 June, 2014; revised 11 August, 2014; accepted 13 August, 2014; published online 19 September, 2014

Keywords: HEK293T cells, knockdown, microRNA, network, SCYL1-BP1
(Yan et al., 2010a). hPirh2 and Mdm2 are important E3 ubiquitin ligases of p53 (Brooks and Gu, 2006; Leng et al., 2003); therefore, SCYL1-BP1 also acts as a co-factor to affect the p53 pathway(Yan et al., 2010b). SCYL1-BP1 gene mutations are associated with premature aging syndromes such as Geroderma Osteodysplasticum(Al-Dosari and Alkuraya, 2009; Hennies et al., 2008). However, the specific molecular mechanism of SCYL1$\mathrm{BP} 1$ and its biological functions in disease progression remain unknown.

MicroRNAs (miRNAs) are a class of endogenous, 19-23 nucleotide, non-coding, single-stranded small RNAs, which are widespread and highly conserved in animals and plants (Bartel, 2009; Esquela-Kerscher and Slack, 2006). Through perfect or partial base-pairing, mainly at the 3 '-untranslated region ( $3^{\prime}$-UTR) of the target gene mRNA, miRNAs can guide the degradation of the target mRNA silencing complex (RISC) and negatively regulate the expression of target mRNA at translational and even the transcriptional level (Forman et al., 2008; Hou et al., 2011; Lytle et al., 2007). MiRNAs can regulate more than $30 \%$ of genes in eukaryotic cells, and participate in multiple regulatory pathways, such as cell proliferation, differentiation, development, aging and apoptosis. Moreover, certain miRNAs suppress or promote tumors in tumorigenesis, making them potential biomarkers for the diagnosis and prognosis oftumors.

Here, using HEK293T cells as an experimental paradigm of transfection, we report that miRNA expression levels were significantly altered when the expression of the SCYL1-BP1 gene was knocked down using RNA interference (RNAi). We used network analysis to functionally characterize the significant impact of potential kernel miRNA-target genes. Importantly, we showed that the expression levels of EEA1, BMPR2 and BRCA2 dramatically decreased when SCYL1-BP1 expression was ablated in HEK293T cells. These data contribute to our understanding of the functions of SCYL1-BP1.

\section{MATERIALS AND METHODS}

Cell culture, transfection and SCYL1-BP1 knockdown by small interfering RNA (siRNA)

Human embryonic kidney cells 293T (HEK293T) were cultured in Dulbecco's Modified Eagle Medium (DMEM; Mediatech Inc., USA) supplemented with $10 \%$ fetal bovine serum (FBS; Biochrom, Germany), $2 \mathrm{mM} \mathrm{L-glutamine,} 100 \mathrm{U} / \mathrm{ml}$ penicillin, and $100 \mu \mathrm{g} / \mathrm{ml}$ streptomycin at $37^{\circ} \mathrm{C}$ in a $5 \% \mathrm{CO}_{2}$ humidified atmosphere. 

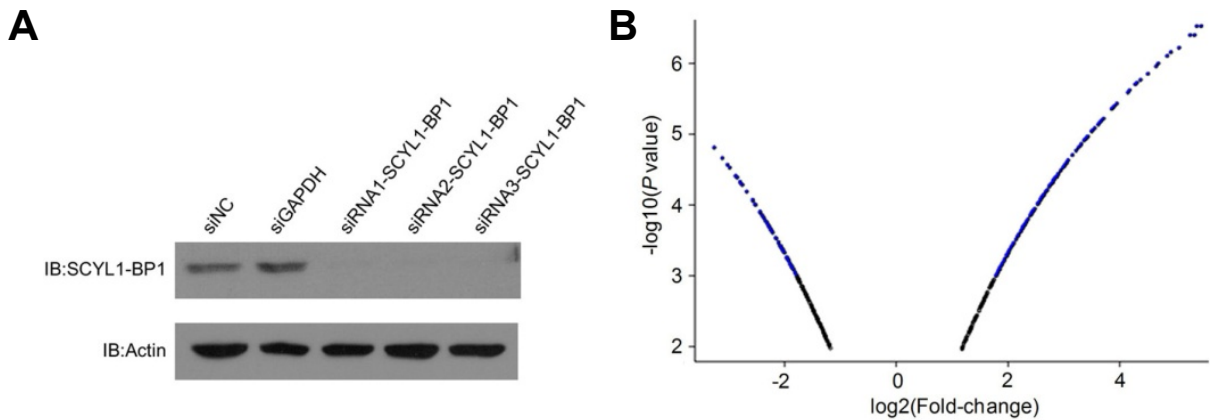

Fig. 1. RNAi could significantly inhibit the expression of endogenous SCYL1-BP1 at the protein level, and altered the distribution of differentially expressed miRNAs. (A) HEK293T cells were transfected with siRNA1-SCYL1-BP1, siRNA2-SCYL1-BP1, siRNA3-SCYL1-BP1, siNegative Control and siGAPDH. The expressions of Actin and SCYL1-BP1 were assayed by Western blotting. (B) The distribution of differentially expressed miRNAs between knockdown and control; a fold-change $>1.5$ and $P<0.001$ were considered to be significant.

All transfection experiments were performed using Lipofectamine 2000 (Invitrogen, USA), following the manufacturer's instructions.

The sequences of the siRNA oligonucleotides numbers 1, 2, and 3 , corresponding to the SCYL1-BP1 mRNA, were GGAGACUAAAGCAGACUAATT, GGCCAGCUUAGACUAUUCATT, and CCCAAAUCAAGAAGGUAAUTT, respectively. A disordered se-quence of the oligonucleotides that has no interfering function in the human genome, UUCUCCGAACGUGUCACGUTT, served as the negative control. Western blotting was used to detect the knockdown efficiency in all cells.

\section{Cell collection and RNA isolation}

The HEK293T cells of SCYL1-BP1 knockdown and control groups were collected and a miRVana PARIS kit (Ambion, USA) was used to extract the total RNA, according to the manufacturer's protocol, and based on the RNase-free DNase I (Promega, USA) to eliminate DNA contamination. The concentration of the RNAs isolated from cells ranged from $1.5-12 \mathrm{ng} / \mu \mathrm{l}$.

MiRNA microarray and data analysis

MiRNA profiles of knockdown and control cells were generated using an Agilent Human miRNA microarray V3 (Agilent Technologies Inc., USA); $60 \mathrm{ng}$ of RNA was labeled and hybridized for each array. Hybridization signals were detected with the Agilent microarray scanner, and the data were extracted using Feature Extraction V10.7 (Agilent Technologies). All raw data were transformed to log2 and their expressions were normalized each by zero mean and unit sample variance.

Using the random variance model $t$-test of the R package, the differential expression miRNAs were calculated between SCYL1-BP1 knockdown and control groups. A fold-change $>1.5$ and $P<0.001$ were considered significant. Furthermore, miRPath (v2.0) was used to classify the miRNA-related pathways $(P<0.001)$ (Vlachos et al., 2012), which were based on experimentally validated data from the TarBase database (v6.0).

Identification of miRNA-target genes and miRNA-target network construction

Three databases were used to predict miRNA-target genes: TarBase (v6.0) (Vergoulis et al., 2012), miRecords (2013) (Xiao et al., 2009) and miRTarBase (2013) (Hsu et al., 2011), which host the largest collection of manually curated experimental data. In this work, we mainly focused on validated target genes; we plan to study the unverified miRNA-target genes in the future.

The weighted miRNA-target network was constructed using
miRNAs and experimentally validated target genes. In the process of network building, miRNAs were weighted by their expression-fold changes $(|\log 2|)$, and target genes were weighted based on the degree distributions. To validate the veracity of a network, all nodes of the network were ranked according to their weights and their similarity was tested (Chen et al., 2013a; Zheng et al., 2011). Thereafter, we obtained deregulated nodes for mapping the consecutive networks. In the weighted miRNAtarget network, the nodes represent miRNAs or genes and the edges represent the connection strength. Then, the simplified miRNA-target network was reconstructed using optimized nodes, which were defined as Degree (D) $\geq 2$ Average (d) from the degree distribution of the network.

Target gene-related protein-protein interaction (PPI) network construction and potential kernel gene selection The MINT database was used to construct PPI networks related to target genes. We first calculated the topological profiles of a PPI network using the ClusterONE algorithm, which was defined as $P$-value $\leq 0.001$, node size $\geq 6$, and network density $\geq 0.05$, and obtained the kernel gene-related clusters from the network. The selected clusters were subsequently reconstructed as a cluster-related PPI network. Next, we calculated the values of Betweenness Centrality (BC), Closeness Centrality (CC), Degree (d) and Topological Coefficient (TC) for screening the potential kernel nodes from the cluster-related PPI network.

Enrichment analysis of target genes

Of the inferred miRNA-target genes, those showing a significant $(P<0.001)$ expression difference between up- and downregulated miRNAs were analyzed for pathways involving these genes using DAVID online analysis (Huang da et al., 2009a; 2009b). Significance analysis was determined when $P$ values were corrected for the false discovery rate (FDR). Gene sets containing fewer than five genes overlapping were removed from the DAVID analysis. In our analysis, GO terms and pathways with an FDR-adjusted $P$-value of less than 0.05 were retained.

\section{Quantification of hub target genes in SCYL1-BP1} knockdown HEK293T cells Quantitative real-time PCR (q-PCR) was performed with SYBR Green PCR Master Mixture (TOYOBO, LTD, Japan) according to the manufacturer's instructions, using a Rotor-Gene 6000 Real-time PCR machine (Corbett Life Science, Australia). Melting curve analysis at the end of the PCR cycles validated the 
A
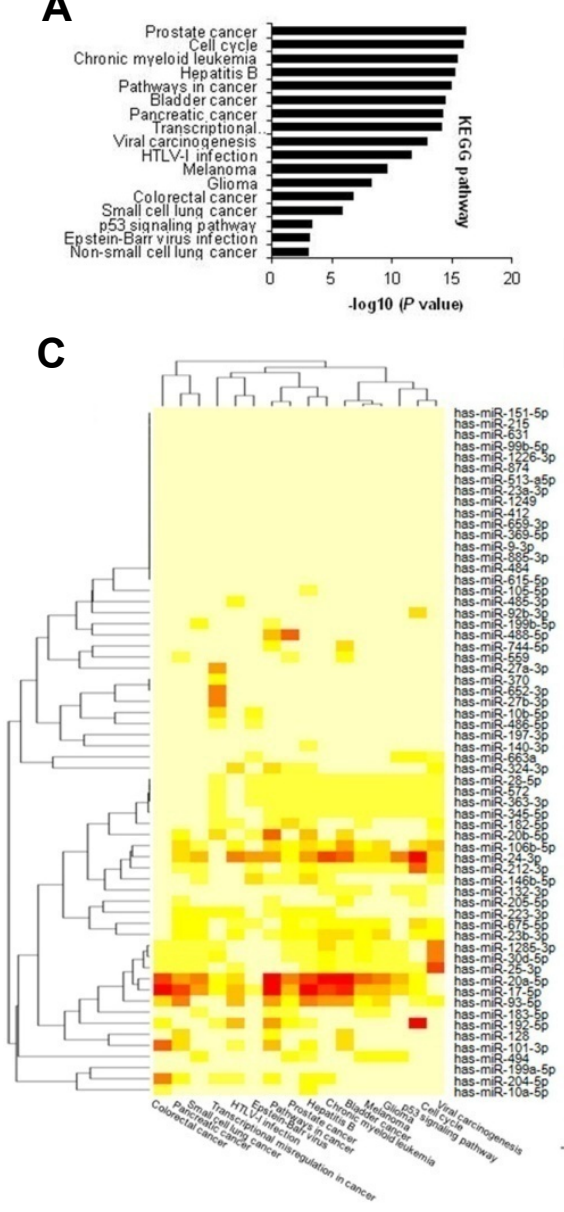

B

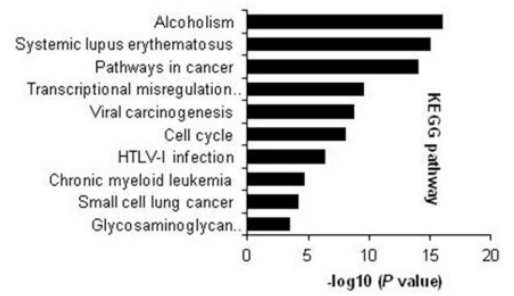

D

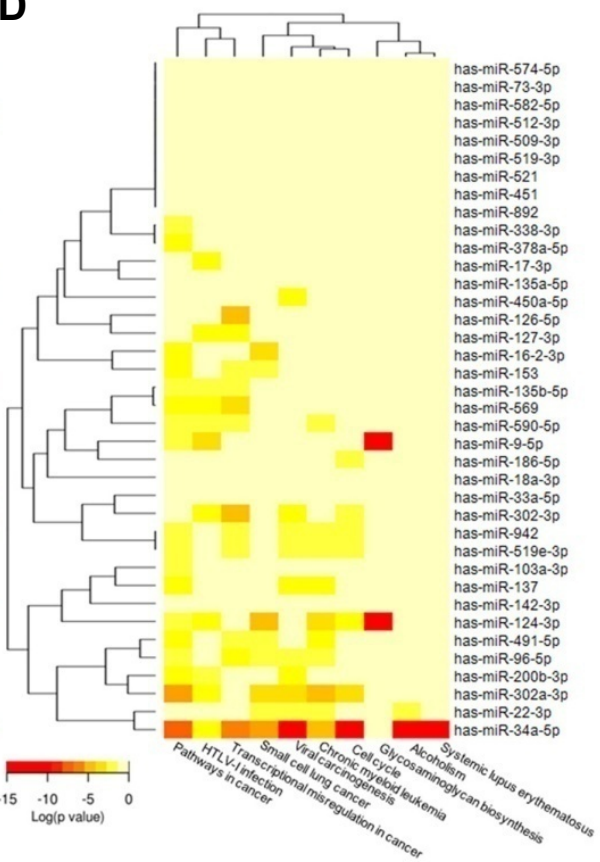

Fig. 2. KEGG pathway analyses of up- and downregulated miRNAs using the miRPath program, and heat maps of miRNAs versus pathways based on their interaction levels. (A) Upregulated miRNA-related KEGG pathways. (B) Downregulated miRNA-related KEGG pathways. (C) Hierarchical cluster and heat map of upregulated miRNAs. (D) Hierarchical cluster and heat map of downregulated miRNAs. Pathways were considered significant at a $P$-value $<0.001$.

specificity of each PCR product. All mRNAs were analyzed in triplicate, and the cycle threshold (Ct) was defined as the number of cycles required for the fluorescent signal to reach the threshold. The levels of mRNAs in cells were calculated using the formula $2 \Delta \mathrm{Ct}$ where $\Delta \mathrm{Ct}=\mathrm{Ct}$ of internal reference $-\mathrm{Ct}$ of target mRNA.

\section{RESULTS}

Differentially expressed miRNAs in SCYL1-BP1 knockdown HEK293T cells

Three small interfering RNA sequences (siRNA1-, siRNA2-, and siRNA3-SCYL1-BP1) and a control sequence (siNC, siGAPDH) were used to perform RNA interference (RNAi) in HEK293T cells. Western blotting validated the knockdown efficiency (Fig. 1A). Compared with the control group, the SCYL1-BP1 expression level was significantly decreased in cells treated with the siRNAs.

The miRNA profiles were calculated using the random module $t$-test of the $\mathrm{R}$ package, to identify differentially expressed miRNAs between the knockdown and control cells. Figure 1B shows that 259 differentially expressed miRNAs were identified between knockdown and control cells, including 168 upregulated and 91 downregulated miRNAs.

To investigate the biological functions of these differentially expressed miRNAs, miRPath (v2.0) analysis was performed to identify miRNA pathways (Vlachos et al., 2012), and significant pathways were determined when $P$-value $<0.001$. The results suggested that 17 pathways are highly associated with 61 upregulated miRNAs, and 10 pathways are associated with 38 downregulated miRNAs (Figs. $2 \mathrm{~A}$ and $2 \mathrm{~B}$ ). The hierarchical cluster and heat map of miRNAs versus pathways were calculated using their interaction levels (Figs. 2C and 2D).

Comparing the miRNA-related pathway terms, seven pathways overlapped between the up- and downregulated miRNAs, which are mainly associated with cell cycle, viral carcinogenesis, chronic myeloid leukemia, and cancer-related pathways. This suggests that these pathways are indispensable for both SCYL1-BP1 knockdown and control HEK293T cells. Strikingly, 10 pathways of upregulated miRNAs and three pathways of downregulated miRNAs were dramatically different. These data suggested that the regulatory mechanism of the upregulated miRNAs may be more complicated, because high expression levels of miRNAs are more likely to inhibit translation or induce target mRNA degradation (Hou et al., 2011). 


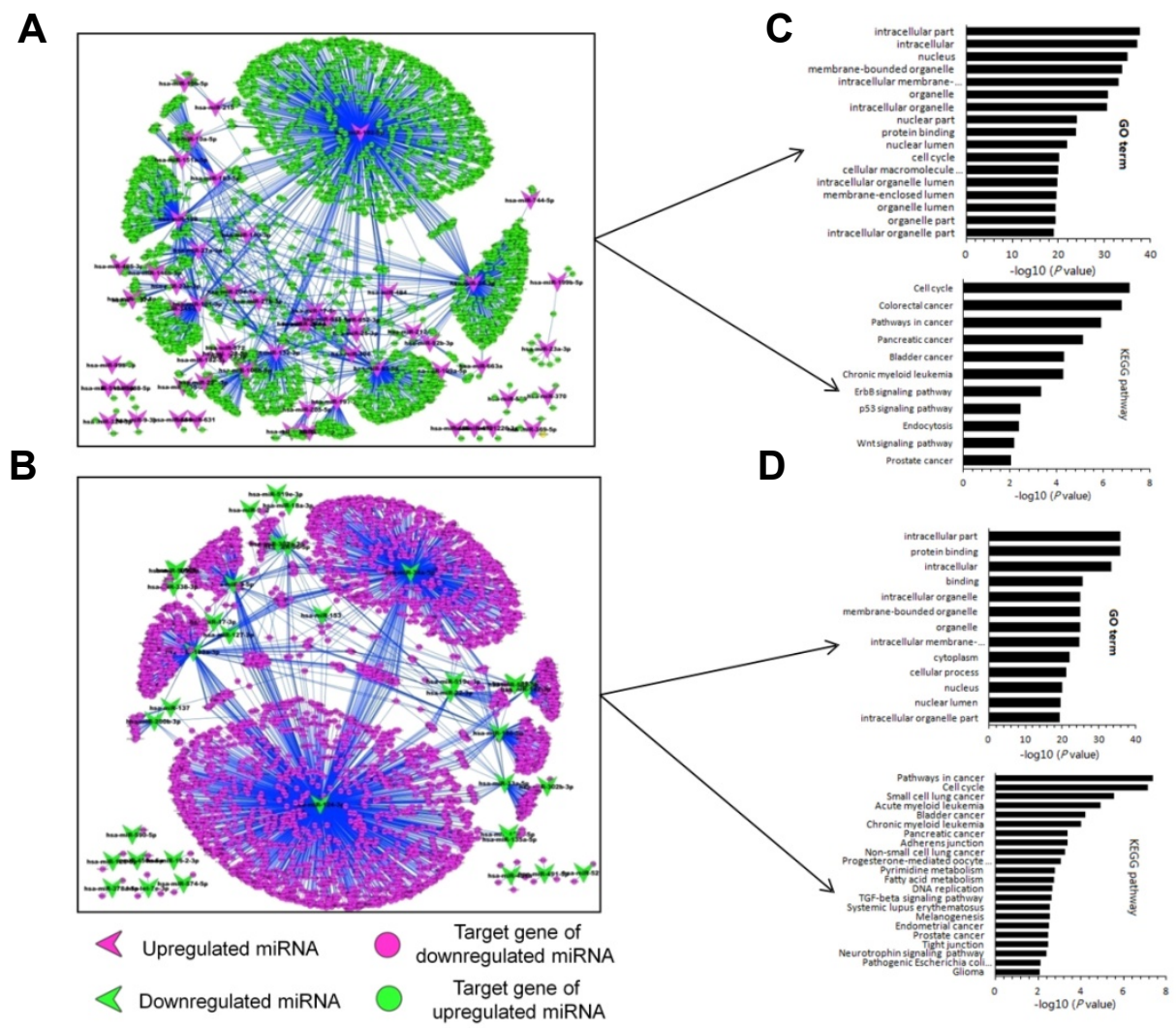

Fig. 3. The differential expression miRNAs and target gene-related networks, and the target genes associated with GO terms and pathways in SCYL1-BP1 knockdown HEK293T cells. (A) Upregulated miRNA target network. (B) Downregulated miRNA-target network. (C) Upregulated miRNAs target gene-related GO terms and pathways. (D) Downregulated miRNAs target gene-related GO terms and pathways.

Overview of miRNA-target networks and enrichment analysis Validated target genes of differentially expressed miRNAs were selected based on TarBase (v6.0), miRecords (2013) and miRTarBase (2013). After redundancy analysis, the final database of miRNA-target genes was built, which included 2887 target genes regulated by 94 upregulated miRNAs and 3288 target genes regulated by 38 downregulated miRNAs.

Using the miRNAs and relative target genes, we built an upregulated miRNA-target network and a downregulated miRNA-target network. As shown in Figs. 3A and 3B, the topological structure of the network is similar to a 'Medusa' model (Guo et al., 2011), which comprises a regulatory core with kernel nodes. It indicates that the hub nodes (miRNAs or target genes) of the network are determinants of the observed gene expression profiles, but the peripheral nodes should be regulated, but are not regulating (Chen et al., 2013b).

To understand these networks holistically, we conducted functional enrichment analysis for the target genes of the networks using DAVID analysis (Huang da et al., 2009a; 2009b). GO terms $(10 \%$ top terms) analysis revealed that the targets of the upregulated miRNA are mainly associated with intracellular, nucleus, protein binding, membrane-bounded organelle, cellular macromolecule metabolic process, cell cycle, enzyme-linked receptor protein signaling pathway, regulation of transcription and cell proliferation (Fig. $3 \mathrm{C}$ ). Similarly, the targets of the downregulated miRNAs are mainly associated with intracellular part, protein binding, intracellular membrane-bounded organelle, cytoplasm and cellular process (Fig. 3D). Importantly, KEGG pathway analysis showed a functional association of upregulated miRNA targets with various cancer-related pathways, such as Colorectal cancer $\left(P=1.62 \times 10^{-7}\right)$, Pathways in cancer $\left(P=1.21 \times 10^{-6}\right)$, Pancreatic cancer $\left(P=7.35 \times 10^{-6}\right)$, Bladder cancer $\left(P=4.91 \times 10^{-6}\right)$, Chronic myeloid leukemia $\left(P=5.13 \times 10^{-5}\right)$, ErbB signaling pathway $\left(P=4.59 \times 10^{-4}\right)$, p53 signaling pathway $\left(P=3.53 \times 10^{-3}\right)$, Endocytosis $\left(P=4.24 \times 10^{-3}\right)$, Wnt signaling pathway $\left(P=6.35 \times 10^{-3}\right)$, as well as Cell cycle $(P$ $\left.=7.32 \times 10^{-8}\right)$ and Prostate cancer $\left(P=9.19 \times 10^{-3}\right)$ (Fig. 3).

Thus, these bioinformatics analyses suggested that ablation of SCYL1-BP1 expression significantly increased the levels of cancer-related miRNAs. The SCYL1-BP1 landscape in the miRNA maps of HEK293T cells may subsequently affect several biological processes, such as cancer progression.

PPI network analysis for target genes of upregulated miRNAs The simplified miRNA-target network was reconstructed using screened nodes, which were defined as Degree (D) $\geq 2$ Average (d) from the degree distribution of the network. As shown in Fig. 4A, 594 target genes overlapped in both simplified networks, which suggest that the common targets play important roles in HEK293T cells. For this reason, we constructed a PPI network for common targets using the MINT database. The PPI network profile is shown in Supplementary Fig. S1A. Using the ClusterONE algorithm (Nepusz et al., 2012), 30 clusters were isolated from the PPI network, which were defined as $P$-value $\leq$ 0.001 , node size $\geq 6$, and network density $\geq 0.05$ (Supplementary Fig. S2; Supplementary Table S1). To estimate the relationship 
Table 1. The distribution of potential kernel nodes from the PPI network and relative qPCR-validated results

\begin{tabular}{|c|c|c|c|c|c|c|c|}
\hline & $\begin{array}{l}\text { Gene } \\
\text { symbol }\end{array}$ & $\begin{array}{c}\text { Closeness } \\
\text { centrality }\end{array}$ & Degree & $\begin{array}{c}\text { Betweenness } \\
\text { centrality }\end{array}$ & $\begin{array}{c}\text { Topological } \\
\text { coefficient }\end{array}$ & $\begin{array}{c}\text { Expression } \\
\text { value } \\
\text { (Knockdown) }\end{array}$ & $\begin{array}{c}\text { Expression } \\
\text { value } \\
\text { (Control) }\end{array}$ \\
\hline \multirow{19}{*}{$\begin{array}{l}\text { Common } \\
\text { targets }\end{array}$} & APPL1 & 0.1554 & 6 & 0.0144 & 0.2361 & 4.6539 & 6.1072 \\
\hline & BCL2 & 0.1770 & 13 & 0.0402 & 0.0905 & 4.4642 & 5.5385 \\
\hline & C8orf33 & 0.1385 & 22 & 0.0605 & 0.0454 & 5.5917 & 7.0576 \\
\hline & ССТ3 & 0.2171 & 3 & 0.0186 & 0.5191 & 8.5699 & 10.7811 \\
\hline & EEA1 & 0.1555 & 9 & 0.0231 & 0.1717 & 1.7756 & 3.3968 \\
\hline & $\mathrm{EZH} 2$ & 0.2235 & 14 & 0.1635 & 0.0855 & 6.6229 & 8.4216 \\
\hline & GRB2 & 0.1965 & 37 & 0.1682 & 0.0327 & 6.1013 & 7.4184 \\
\hline & HIPK3 & 0.1346 & 13 & 0.0434 & 0.1025 & 2.6519 & 3.7642 \\
\hline & ITGB1 & 0.1862 & 11 & 0.0687 & 0.1034 & 5.7594 & 7.1643 \\
\hline & MAPK14 & 0.1986 & 18 & 0.2012 & 0.0784 & 4.7795 & 5.7354 \\
\hline & MCL1 & 0.1744 & 10 & 0.0370 & 0.1333 & 5.5614 & 6.8035 \\
\hline & MCM3 & 0.1975 & 7 & 0.0374 & 0.2258 & 7.7238 & 9.7779 \\
\hline & PCNA & 0.1644 & 20 & 0.052 & 0.0636 & 6.6981 & 8.8594 \\
\hline & PDLIM7 & 0.1858 & 3 & 0.0494 & 0.4973 & 4.4889 & 5.3724 \\
\hline & PPP1R12A & 0.1968 & 4 & 0.0188 & 0.2500 & 4.2588 & 5.5308 \\
\hline & PRMT5 & 0.2369 & 36 & 0.2195 & 0.0286 & 5.8914 & 7.3711 \\
\hline & SMAD4 & 0.1900 & 35 & 0.0953 & 0.0285 & 3.4742 & 4.7586 \\
\hline & SPRY2 & 0.1977 & 17 & 0.0704 & 0.0617 & 5.3249 & 6.4559 \\
\hline & VCL & 0.1965 & 3 & 0.0102 & 0.3333 & 5.6283 & 6.7397 \\
\hline \multirow{11}{*}{$\begin{array}{l}\text { Unique } \\
\text { targets }\end{array}$} & BMPR2 & 0.1661 & 3 & 0.0559 & 0.3333 & 2.4658 & 4.2482 \\
\hline & BNIP2 & 0.1820 & 3 & 0.0334 & 0.3333 & 4.4202 & 5.8564 \\
\hline & BRCA1 & 0.1519 & 14 & 0.0481 & 0.0904 & 4.8327 & 6.4537 \\
\hline & BRCA2 & 0.1517 & 11 & 0.0436 & 0.1111 & 1.0285 & 3.0866 \\
\hline & DDX5 & 0.1914 & 16 & 0.0643 & 0.0984 & 6.875 & 9.5271 \\
\hline & GNB5 & 0.1513 & 4 & 0.0283 & 0.3125 & 3.7189 & 4.6893 \\
\hline & IRAK1 & 0.6400 & 7 & 0.4277 & 0.2527 & 6.5358 & 8.2885 \\
\hline & KIF23 & 0.1826 & 5 & 0.0477 & 0.2000 & 6.3873 & 8.151 \\
\hline & RBL2 & 0.1874 & 5 & 0.0754 & 0.2303 & 3.6676 & 4.9137 \\
\hline & STAU1 & 0.1293 & 3 & 0.0231 & 0.3333 & 6.2764 & 7.9761 \\
\hline & UBE2D1 & 0.1886 & 11 & 0.1947 & 0.1157 & 4.5769 & 5.9081 \\
\hline
\end{tabular}

among these clusters, the cluster-related PPI network was reconstructed by these 30 clusters. As shown in Fig. 4B, the topological profile revealed that the correlation of the clusters was very tight. Similarly, the PPI network for unique targets (no including overlapped targets) of upregulated miRNAs was also constructed (Supplementary Fig. S1B), and 28 clusters ( $P$-value $\leq 0.001$ ) were isolated from the network (Supplementary Fig. S3; Supplementary Table 2). The reconstructed cluster-related PPI network is shown in Fig. 4C.

To screen the potential kernel nodes from the cluster-related PPI networks, we calculated the BC, CC, (d) and TC for each node in the network. The selected degree was defined as $d \geq 2$. The other parameters were defined as $\mathrm{BC} \geq$ Average (BC), CC $\geq$ Average (CC), and TC $\geq$ Average (TC), respectively. Finally, 19 kernel nodes were screened from the common target-related PPI network and 11 kernel nodes were selected from unique target-related PPI network (Table 1).

\section{Establishing and validating the potential kernel genes}

To explore whether these hub nodes are potential kernel genes in predicting the progression of network, we performed qPCR to measure the expression levels of 30 genes in SCYL1-BP1 knockdown and control HEK293T cells (Table 1).

As shown in Fig. $5 \mathrm{~A}$, the total targets set $(n=30)$ included common targets $(n=19)$ and upregulated miRNA unique targets $(n=11)$. At the transcript expression level, statistical significance was noted for the total targets $(P=0.001)$, common tar- gets $(P=0.009)$ and unique targets $(P=0.025)$. Their average levels were significantly downregulated in SCYL1-BP1 knockdown compared with the control HEK293T cells. Remarkably, the expression levels of EEA1, BMPR2 and BRCA2 are highlighted, with prominent coverage to other potential kernel genes with much higher expression levels than other potential kernel genes (Fig. 5B).These data suggest that the diminished expression level of kernel genes in HEK293T cells, especially EEA1, $B M P R 2$ and BRCA2, may represent one of the molecular mechanisms underlying the effects of global loss of SCYL1-BP1.

\section{DISCUSSION}

The SCYL1-BP1 interacts with several proteins (Yan et al., 2010a; Zhang et al., 2005). However, the specific function and mechanism of SCYL1-BP1 remain unclear. As a class of gene regulators, miRNAs have an important combinatorial hallmark in the gene regulation process (Chen et al., 2013a). Generally, a given miRNA may have multiple different mRNA targets; a given target might also be targeted by multiple miRNAs (Krek et al., 2005; van Iterson et al., 2013). In this study, we generated miRNA expression maps of SCYL1-BP1 knockdown and control in HEK293T cells using an miRNA microarray, and observed the miRNAs levels were significantly changed by comparison. Subsequently, the networks of up- and downregulated miRNA-target were constructed. The topological structures were similar to the 'Medusa' model, which implied that the hub nodes are determi- 


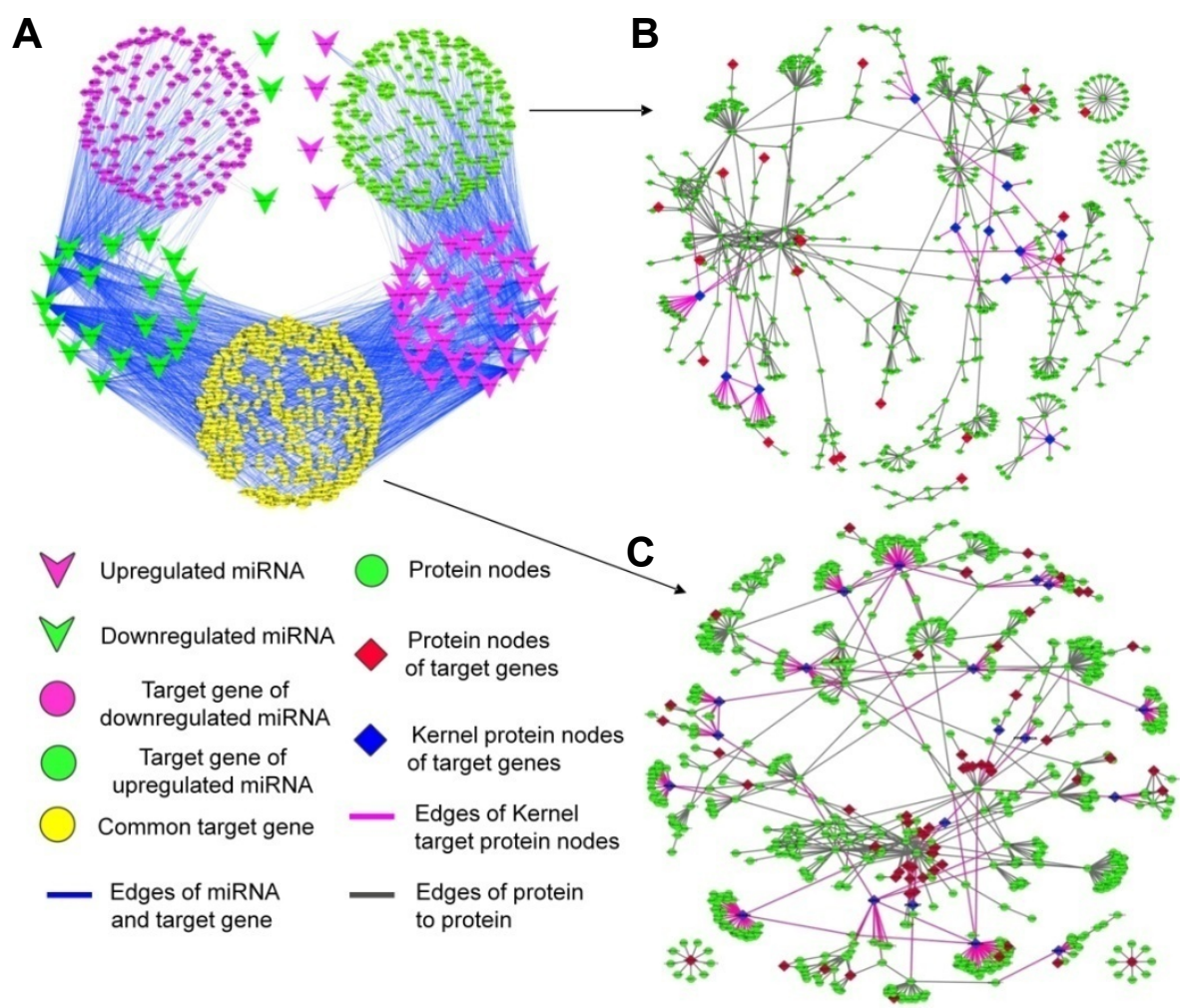

Fig. 4. Merged simplified miRNA-target network and PPI network of common targets and upregulated miRNAs unique targets. (A) Merged simplified network of up- and downregulated miRNA-target networks. (B) Unique targets of upregulated miRNA-related PPI networks. (C) Common targetrelated PPI networks. Note that the PPI networks of $(B)$ and $(C)$ were reconstructed by significant clusters of individual original PPI networks. The blue diamond nodes represent hub nodes based on topological analysis, including $B C>0.01, C C>0.001, T C>0.01$, and $B C>0.01$.

A

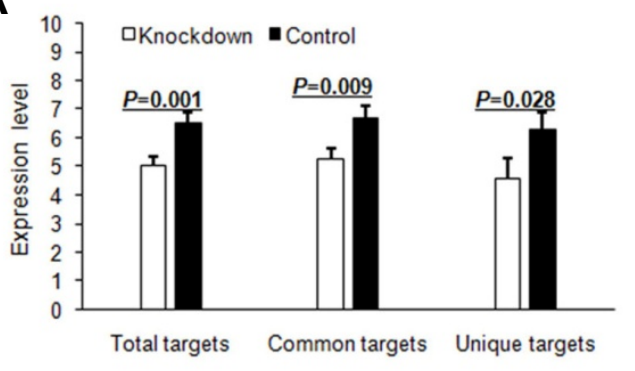

B

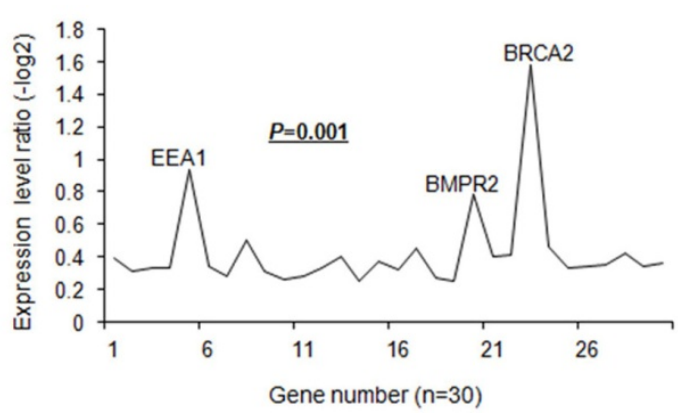

Fig. 5. qPCR validation of the potential kernel target genes. (A) The mRNA levels of total targets $(n=30)$, common targets $(n=19)$ and upregulated miRNA unique targets $(\mathrm{n}=11)$. Illustrated $P$-values are based on pair-wise comparisons using Mann-Whitney $U$ tests. (B) The potential target genes $(n=30)$ expression level ratio of knockdown/Control $(-\log 2)$; EEA1, BMPR2 and BRCA2 levels are dramatically decreased in SCYL1-BP1 knockdown cells.

nants of the observed gene expression profiles (Chen et al., 2012; Zheng et al., 2011).

GO terms and pathway analysis revealed that the upregulated miRNAs and target genes are mainly associated with the cell cycle, p53 Signaling Pathway and cancer-related pathways. The imbalance of G1/S and G2/M phases of the cell cycle is associated with dysfunction in hepatocarcinoma (Spaziani et al., 2006), while the p53 signaling pathway is highly correlated with the pathogenesis of numerous cancers (Stegh, 2013). This implies that ablation of SCYL1-BP1 expression significantly increases the levels of cancer-related miRNAs, which then affect the expressions of their related target genes and transform the original progressions of the cell cycle and p53 signaling pathway in cells.

To determine the biological consequence of ablating SCYL1BP1 expression in cells, we first simplified the miRNA-target network and obtained 594 overlapped target genes between upand downregulated miRNA-target networks. Next, we constructed the common target-related PPI network and upregulated 
miRNAs' unique target-related PPI network, respectively. Finally, 30 nodes representing potential kernel genes were screened from the networks, which might play important roles in biological progression in cells. The qPCR results showed that the expressions of the kernel genes were significantly downregulated in SCYL1-BP1 knockdown cells. Importantly, the mRNA expression levels of EEA1, BMPR2 and BRCA2 were very prominent in the total kernel genes. Thus, these data highlight a potential molecular mechanism of decreased kernel genes levels mediated by the loss of SCYL1-BP1.

EEA1 is a membrane-tethering factor required for the fusion and maturation of early endosomes in endocytosis, which plays a crucial role in the tethering process leading to homotypic endosome fusion (Bergeland et al., 2008). EEA1 is considered a new substrate of $p 97$, and p97 may regulate the oligomeric state of EEA1 to influence its membrane-tethering function, which in turn affects the size of early endosomes (Ramanathan and Ye, 2012). Beas et al. demonstrated that the heterotrimeric $G$ protein Gas facilitates dissociation of EEA1 from membranes. This is a key step in EEA1 endosome maturation, which limits EGFR signaling from EEA1 endosomes, and then inhibits cell proliferation (Beas et al., 2012). In cells, Rab5 and its effector EEA1 associate with membranes of EEA1 endosomes, which is associated with the fusion of endosomes (Poteryaev et al., 2012). Moreover, endogenous EEA1 is a ubiquitin-regulated factor, and may be modified via an E3 independent ubiquitin conjugation mechanism in cells. Furthermore, ubiquitination may significantly alter EEA1 activity to influence endocytosis (Ramanathan et al., 2013).

BMPR2, a member of the bone morphogenetic protein (BMP) receptor family of transmembrane serine/threonine kinases, plays a critical role in the regulation of genomic integrity in pulmonary endothelial cells (Li et al., 2014). Decreased BMPR2 expression can enhance GM-CSF (granulocyte macrophage colony-stimulating factor) mRNA translation, which increases inflammatory cell recruitment and exacerbates idiopathic pulmonary arterial hypertension (PAH)(Sawada et al., 2014). BMPR2 downregulation is also likely to promote vascular smooth muscle cell proliferation and restenosis, which represent a new clue for the early diagnosis of carotid restenosis (Luo et al., 2013). In human adipose-derived mesenchymal stem cells, BMPR2 is targeted by miR-100 and may play a negative role in osteogenic differentiation, which is important for improving the treatment of bone-related diseases (Zeng et al., 2012).

BRCA2, breast cancer susceptibility 2, is a well-known tumor suppressor gene that has been linked to defined human cancer syndromes (Karami and Mehdipour, 2013). Previous studies demonstrated that BRCA2 mutation or deficiency is linked to many human cancers (Birkbak et al., 2013; King et al., 2003; Norquist et al., 2010; Venkitaraman, 2002; Yang et al., 2011). In particular, p53 mutations may have a synergistic effect on tumorigenesis in BRCA2-associated cancers (Shive et al., 2014). In cells, BRCA2 overexpression is associated with poor outcome and correlates with a high proliferation rate (Magwood et al., 2012). Furthermore, BRCA2 suppresses homologous recombination, reduces RAD51 foci formation and inactivates p53 function (Magwood et al., 2012). On the other hand, downregulation of BRCA2 expression has been suggested as a biomarker for docetaxel treatment in breast cancer patients (Egawa et al., 2001). Interestingly, BRCA2 knockdown has been proposed to have a potential therapeutic benefit in multidrug treatment of human tumors (Rytelewski et al., 2013).

In conclusion, diminished expression of SCYL1-BP1 dramatically reduced the expression levels of EEA1, BMPR2 and $B R C A 2$. SCYL1-BP1 is associated with wrinkly skin and osteo- porosis in premature aging syndromes (Al-Dosari and Alkuraya, 2009; Hennies et al., 2008). Furthermore, it also acts as a ubiquitination substrate of hPirh2 and induces Mdm2 selfubiquitination (Yan et al., 2010a). Interestingly, EEA1 is associated with the ubiquitination pathway, BMPR2 is a BMP receptor, and BRCA2 is a tumor suppressor gene. Thus we infer that SCYL1-BP1 contributes to ubiquitination and disease-related biological processes. However, when SCYL1-BP1 is mutated or its expression ablated, the expression levels of EEA1, BMPR2 and BRCA2 are dramatically decreased, which would further interfere with the normal ubiquitination pathway and lead to associated diseases or tumorigenesis. Our data provide a new clue to investigate the molecular mechanisms of SCYL1-BP1.

Note: Supplementary information is available on the Molecules and Cells website (www.molcells.org).

\section{ACKNOWLEDGMENTS}

This work was supported by the National Key Basic Research Program of China (Nos. 2013CB531603 and 2010CB912602) to $\mathrm{K}$ Huo.The Scheming Project of Shanghai Municipal Education Commission (2012JW25), the Leading Project of Integrated Traditional and Western Medicine of Shanghai University of Traditional Chinese Medicine (2013), and the Key Science Foundation of Anhui Province (KJ2012A260) to QL Chen.

\section{REFERENCES}

Al-Dosari, M., and Alkuraya, F.S. (2009). A novel missense mutation in SCYL1BP1 produces geroderma osteodysplastica phenotype indistinguishable from that caused by nullimorphic mutations. Am. J. Med. Genet. A 149A, 2093-2098.

Bartel, D.P. (2009). MicroRNAs: target recognition and regulatory functions. Cell 136, 215-233.

Beas, A.O., Taupin, V., Teodorof, C., Nguyen, L.T., Garcia-Marcos, M., and Farquhar, M.G. (2012). Gas promotes EEA1 endosome maturation and shuts down proliferative signaling through interaction with GIV (Girdin). Mol. Biol. Cell 23, 4623-4634.

Bergeland, T., Haugen, L., Landsverk, O.J., Stenmark, H., and Bakke, O. (2008). Cell-cycle-dependent binding kinetics for the early endosomal tethering factor EEA1. EMBO Rep. 9, 171-178.

Birkbak, N.J., Kochupurakkal, B., Izarzugaza, J.M., Eklund, A.C., Li, Y., Liu, J., Szallasi, Z., Matulonis, U.A., Richardson, A.L., Iglehart, J.D., et al. (2013). Tumor mutation burden forecasts outcome in ovarian cancer with BRCA1 or BRCA2 mutations. PLoS One 8, e80023.

Brooks, C.L., and Gu, W. (2006). p53 ubiquitination: Mdm2 and beyond. Mol. Cell 21, 307-315.

Chen, L., Liu, R., Liu, Z.P., Li, M., and Aihara, K. (2012). Detecting early-warning signals for sudden deterioration of complex diseases by dynamical network biomarkers. Sci. Rep. 2, 342.

Chen, Q.-L., Lu, Y.-Y., Zhang, G.-B., Song, Y.-N., Zhou, Q.-M., Hui Zhang, Zhang, W., and Su, S.-B. (2013a). Progression from excessive to deficient syndromes in chronic hepatitis $\mathrm{B}$ : adynamical network analysis of miRNA array data. Evid. Based Complement. Alternat. Med. 2013, 945245

Chen, Q.L., Lu, Y.Y., Zhang, G.B., Song, Y.N., Zhou, Q.M., Zhang, H. Zhang, W., Tang, X.S., and Su, S.B. (2013b). Characteristic analysis from excessive to deficient syndromes in hepatocarcinoma underlying miRNA array data. Evid. Based Complement. Alternat. Med. 2013, 324636

Di, Y., Li, J., Fang, J., Xu, Z., He, X., Zhang, F., Ling, J., Li, X., Xu, D., $\mathrm{Li}$, L., et al. (2003). Cloning and characterization of a novel gene which encodes a protein interacting with the mitosis-associated kinase-like protein NTKL. J. Hum. Genet. 48, 315-321.

Egawa, C., Miyoshi, Y., Takamura, Y., Taguchi, T., Tamaki, Y., and Noguchi, S. (2001). Decreased expression of BRCA2 mRNA predicts favorable response to docetaxel in breast cancer. Int. J. Cancer 95, 255-259.

Esquela-Kerscher, A., and Slack, F.J. (2006). Oncomirs - microRNAs with a role in cancer. Nat. Rev. Cancer 6, 259-269. 
Forman, J.J., Legesse-Miller, A., and Coller, H.A. (2008). A search for conserved sequences in coding regions reveals that the let-7 microRNA targets Dicer within its coding sequence. Proc. Natl. Acad. Sci. USA 105, $14879-14884$.

Guo, Y., Feng, Y., Trivedi, N.S., and Huang, S. (2011). Medusa structure of the gene regulatory network: dominance of transcription factors in cancer subtype classification. Exp. Biol. Med. (Maywood) 236, 628-636.

Hennies, H.C., Kornak, U., Zhang, H., Egerer, J., Zhang, X., Seifert, W., Kuhnisch, J., Budde, B., Natebus, M., Brancati, F., et al (2008). Gerodermia osteodysplastica is caused by mutations in SCYL1BP1, a Rab-6 interacting golgin. Nat. Genet. 40, 1410 1412.

Hou, J., Lin, L., Zhou, W., Wang, Z., Ding, G., Dong, Q., Qin, L., Wu X., Zheng, Y., Yang, Y., et al. (2011). Identification of miRNomes in human liver and hepatocellular carcinoma reveals miR-199a/b$3 p$ as therapeutic target for hepatocellular carcinoma. Cancer Cell 19, 232-243.

Hsu, S.D., Lin, F.M., Wu, W.Y., Liang, C., Huang, W.C., Chan, W.L., Tsai, W.T., Chen, G.Z., Lee, C.J., Chiu, C.M., et al. (2011). miRTarBase: a database curates experimentally validated microRNA-target interactions. Nucleic Acids Res. 39, D163-169.

Huang da, W., Sherman, B.T., and Lempicki, R.A. (2009a). Bioinformatics enrichment tools: paths toward the comprehensive functional analysis of large gene lists. Nucleic Acids Res. 37, 1-13.

Huang da, W., Sherman, B.T., and Lempicki, R.A. (2009b). Systematic and integrative analysis of large gene lists using DAVID bioinformatics resources. Nat. Protoc. 4, 44-57.

Karami, F., and Mehdipour, P. (2013). A comprehensive focus on global spectrum of BRCA1 and BRCA2 mutations in breast cancer. Biomed. Res. Int. 2013, 928562.

King, M.C., Marks, J.H., and Mandell, J.B. (2003). Breast and ovarian cancer risks due to inherited mutations in BRCA1 and BRCA2. Science 302, 643-646.

Krek, A., Grun, D., Poy, M.N., Wolf, R., Rosenberg, L., Epstein, E.J., MacMenamin, P., da Piedade, I., Gunsalus, K.C., Stoffel, M., et al. (2005). Combinatorial microRNA target predictions. Nat. Genet 37, 495-500.

Leng, R.P., Lin, Y., Ma, W., Wu, H., Lemmers, B., Chung, S., Parant, J.M., Lozano, G., Hakem, R., and Benchimol, S. (2003). Pirh2, a p53-induced ubiquitin-protein ligase, promotes p53 degradation Cell 112, 779-791.

Li, M., Vattulainen, S., Aho, J., Orcholski, M., Rojas, V., Yuan, K., Helenius, M., Taimen, P., Myllykangas, S., De Jesus Perez, V., et al. (2014). Loss of bone morphogenetic protein receptor 2 is associated with abnormal DNA repair in pulmonary arterial hypertension. Am. J. Respir. Cell Mol. Biol.50, 1118-1128

Luo, T., Cui, S., Bian, C., and Yu, X. (2013). Crosstalk between TGFbeta/Smad3 and BMP/BMPR2 signaling pathways via miR-17-92 cluster in carotid artery restenosis. Mol. Cell. Biochem.389, 169176

Lytle, J.R., Yario, T.A., and Steitz, J.A. (2007). Target mRNAs are repressed as efficiently by microRNA-binding sites in the 5' UTR as in the 3' UTR. Proc. Natl. Acad. Sci. USA 104, 9667-9672.

Magwood, A.C., Mundia, M.M., and Baker, M.D. (2012). High levels of wild-type BRCA2 suppress homologous recombination. J. Mol. Biol. 421, 38-53.

Nepusz, T., Yu, H., and Paccanaro, A. (2012). Detecting overlapping protein complexes in protein-protein interaction networks. Nat Methods 9, 471-472.

Norquist, B.M., Garcia, R.L., Allison, K.H., Jokinen, C.H., Kernochan, L.E., Pizzi, C.C., Barrow, B.J., Goff, B.A., and Swisher, E.M (2010). The molecular pathogenesis of hereditary ovarian carcinoma: alterations in the tubal epithelium of women with BRCA1 and BRCA2 mutations. Cancer 116, 5261-5271.

Poteryaev, D., Datta, S., Ackema, K., Zerial, M., and Spang, A (2012). Identification of the switch in early-to-late endosome transition. Cell 141, 497-508.

Ramanathan, H.N., and Ye, Y. (2012). The p97 ATPase associates with EEA1 to regulate the size of early endosomes. Cell Res. 22 346-359.

Ramanathan, H.N., Zhang, G., and Ye, Y. (2013). Monoubiquitination of EEA1 regulates endosome fusion and trafficking. Cell Biosci. 3 24

Rytelewski, M., Ferguson, P.J., Maleki Vareki, S., Figueredo, R., Vincent, M., and Koropatnick, J. (2013). Inhibition of BRCA2 and thymidylate synthase creates multidrug sensitive tumor cells via the induction of combined "Complementary Lethality". Mol. Ther Nucleic Acids 2, e78.

Sawada, H., Saito, T., Nickel, N.P., Alastalo, T.P., Glotzbach, J.P., Chan, R., Haghighat, L., Fuchs, G., Januszyk, M., Cao, A., et al. (2014). Reduced BMPR2 expression induces GM-CSF translation and macrophage recruitment in humans and mice to exacerbate pulmonary hypertension. J. Exp. Med.211, 263-280.

Shive, H.R., West, R.R., Embree, L.J., Golden, C.D., and Hickstein D.D. (2014). brca2 and tp53 collaborate in tumorigenesis in zebrafish. PLoS One 9, e87177.

Spaziani, A., Alisi, A., Sanna, D., and Balsano, C. (2006). Role of p38 MAPK and RNA-dependent protein kinase (PKR) in hepatitis $C$ virus core-dependent nuclear delocalization of cyclin B1. J. Biol. Chem. 281, 10983-10989.

Stegh, A.H. (2013). Targeting the p53 signaling pathway in cancer therapy - the promises, challenges and perils. Exp. Opin. Ther. Targets 16, 67-83.

van Iterson, M., Bervoets, S., de Meijer, E.J., Buermans, H.P., tHoen, P.A., Menezes, R.X., and Boer, J.M. (2013). Integrated analysis of microRNA and mRNA expression: adding biological significance to microRNA target predictions. Nucleic Acids Res. 41, e146.

Venkitaraman, A.R. (2002). Cancer susceptibility and the functions of BRCA1 and BRCA2. Cell 108, 171-182.

Vergoulis, T., Vlachos, I.S., Alexiou, P., Georgakilas, G., Maragkakis, M., Reczko, M., Gerangelos, S., Koziris, N., Dalamagas, T., and Hatzigeorgiou, A.G. (2012). TarBase 6.0: capturing the exponential growth of miRNA targets with experimental support. Nucleic Acids Res. 40, D222-229.

Vlachos, I.S., Kostoulas, N., Vergoulis, T., Georgakilas, G., Reczko, M., Maragkakis, M., Paraskevopoulou, M.D., Prionidis, K., Dalamagas, T., and Hatzigeorgiou, A.G. (2012). DIANA miRPath v.2.0: investigating the combinatorial effect of microRNAs in pathways. Nucleic Acids Res. 40, W498-504.

Xiao, F., Zuo, Z., Cai, G., Kang, S., Gao, X., and Li, T. (2009). miRecords: an integrated resource for microRNA-target interactions. Nucleic Acids Res. 37, D105-110.

Yan, J., Zhang, D., Di, Y., Shi, H., Rao, H., and Huo, K. (2010a). A newly identified Pirh2 substrate SCYL1-BP1 can bind to MDM2 and accelerate MDM2 self-ubiquitination. FEBS Lett. 584, 32753278

Yan, J., Di, Y., Shi, H., Rao, H., and Huo, K. (2010b). Overexpression of SCYL1-BP1 stabilizes functional p53 by suppressing MDM2mediated ubiquitination. FEBS Lett. 584, 4319-4324.

Yang, D., Khan, S., Sun, Y., Hess, K., Shmulevich, I., Sood, A.K., and Zhang, W. (2011). Association of BRCA1 and BRCA2 mutations with survival, chemotherapy sensitivity, and gene mutator phenotype in patients with ovarian cancer. JAMA 306, 1557-1565.

Zeng, Y., Qu, X., Li, H., Huang, S., Wang, S., Xu, Q., Lin, R., Han, Q. Li, J., and Zhao, R.C. (2012). MicroRNA-100 regulates osteogenic differentiation of human adipose-derived mesenchymal stem cells by targeting BMPR2. FEBS Lett. 586 , 2375-2381.

Zhang, L., Li, J., Wang, C., Ma, Y., and Huo, K. (2005). A new human gene hNTKL-BP1 interacts with hPirh2. Biochem. Biophys. Res. Commun. 330, 293-297.

Zheng, S., Tansey, W.P., Hiebert, S.W., and Zhao, Z. (2011). Integrative network analysis identifies key genes and pathways in the progression of hepatitis $C$ virus induced hepatocellular carcinoma BMC Med. Genomics 4, 62 